\title{
New structural analogues of curcumin exhibit potent growth suppressive activity in human colorectal carcinoma cells Ling Cen ${ }^{1}$, Brian Hutzen ${ }^{1,2}$, Sarah Ball1,2, Stephanie DeAngelis ${ }^{1}$, Chun- Liang Chen ${ }^{1}$, James R Fuchs ${ }^{3}$, Chenglong $\mathrm{Li}^{3}$, Pui-Kai $\mathrm{Li}^{3}$ and Jiayuh Lin*1,2,4,5
}

\begin{abstract}
Address: ${ }^{1}$ Department of Pediatrics, The Ohio State University, Columbus, Ohio, 43210, USA, ${ }^{2}$ Molecular Cellular and Developmental Biology Program, The Ohio State University, Columbus, Ohio, 43210, USA, 3 Division of Medicinal Chemistry and Pharmacognosy, College of Pharmacy, The Ohio State University, Columbus, Ohio, 43210, USA, ${ }^{4}$ Experimental Therapeutics Program, The Ohio State University Comprehensive Cancer Center, College of Medicine, The Ohio State University, Columbus, Ohio, 43210, USA and ${ }^{5}$ Center for Childhood Cancer, The Research Institute at Nationwide Children's Hospital, Department of Pediatrics, College of Medicine, The Ohio State University, 700 Children's Drive, Columbus, $\mathrm{OH} 43205$, USA

Email: Ling Cen - cen.ling@mayo.edu; Brian Hutzen - Hutzen.1@osu.edu; Sarah Ball - Ball.1766@osu.edu; Stephanie DeAngelis - Stephanie.DeAngelis@nationwidechildrens.org; Chun-Liang Chen - Chenc@pediatrics.ohio-state.edu; James R Fuchs - fuchs@pharmacy.ohio-state.edu; Chenglong Li - cli@pharmacy.ohio-state.edu; Pui-Kai Li - li.27@osu.edu; Jiayuh Lin* - lin.674@osu.edu

* Corresponding author
\end{abstract}

Published: 30 March 2009

BMC Cancer 2009, 9:99 doi:10.1186/147/-2407-9-99
Received: 7 September 2008

Accepted: 30 March 2009

This article is available from: http://www.biomedcentral.com/l47I-2407/9/99

(C) 2009 Cen et al; licensee BioMed Central Ltd.

This is an Open Access article distributed under the terms of the Creative Commons Attribution License (http://creativecommons.org/licenses/by/2.0), which permits unrestricted use, distribution, and reproduction in any medium, provided the original work is properly cited.

\begin{abstract}
Background: Colorectal carcinoma is one of the major causes of morbidity and mortality in the Western World. Novel therapeutic approaches are needed for colorectal carcinoma. Curcumin, the active component and yellow pigment of turmeric, has been reported to have several anticancer activities including anti-proliferation, anti-invasion, and anti-angiogenesis. Clinical trials have suggested that curcumin may serve as a potential preventive or therapeutic agent for colorectal cancer.

Methods: We compared the inhibitory effects of curcumin and novel structural analogues, GOY030, FLLL-I I, and FLLL-12, in three independent human colorectal cancer cell lines, SW480, HT29, and HCTII6. MTT cell viability assay was used to examine the cell viability/proliferation and western blots were used to determine the level of PARP cleavages. Half-Maximal inhibitory concentrations $\left(\mathrm{IC}_{50}\right)$ were calculated using Sigma Plot 9.0 software.

Results: Curcumin inhibited cell viability in all three of the human colorectal cancer cell lines studied with $\mathrm{IC}_{50}$ values ranging between $10.26 \mu \mathrm{M}$ and I3.3I $\mu \mathrm{M}$. GO-Y030, FLLL-II, and FLLL12 were more potent than curcumin in the inhibition of cell viability in these three human colorectal cancer cell lines with $\mathrm{IC}_{50}$ values ranging between $0.5 \mathrm{I} \mu \mathrm{M}$ and $4.48 \mu \mathrm{M}$. In addition, FLLL-II and FLLL- 12 exhibit low toxicity to WI-38 normal human lung fibroblasts with an IC-50 value greater than I,000 $\mu \mathrm{M}$. GO-Y030, FLLL-I I, and FLLL-I 2 are also more potent than curcumin in the induction of apoptosis, as evidenced by cleaved PARP and cleaved caspase- 3 in all three human colorectal cancer cell lines studied.

Conclusion: The results indicate that the three curcumin analogues studied exhibit more potent inhibitory activity than curcumin in human colorectal cancer cells. Thus, they may have translational potential as chemopreventive or therapeutic agents for colorectal carcinoma.
\end{abstract}




\section{Background}

In the United States, colorectal cancer is the third most frequently occurring cancer in both sexes and overall is the second leading cause of cancer deaths. The lifetime probability of developing colorectal cancer is about $5 \%$ in the United States. Despite advances in the treatment of colorectal cancer, the five-year survival rate has only increased to $65 \%$ [1]. Hence, better approaches for the prevention and treatment of colorectal cancer are needed.

Curcumin has been shown to protect against carcinogenesis and to prevent tumor formation and development in several cancer types. It has also been shown to suppress angiogenesis and metastasis in a variety of animal tumor models [2-6]. Curcumin is a bioactive component found in the rhizome of the perennial herb Curcuma longa. A polyphenolic compound with intense yellow coloring, curcumin has been part of therapeutic preparations for centuries due to its wide spectrum of beneficial activities and its safety in relatively large doses [7]. Extensive research has indicated that the complex chemistry of curcumin allows it to influence multiple cell signaling pathways, giving it anti-inflammatory, antioxidant, chemopreventive, and chemotherapeutic properties in addition to many others [8]. The anti-carcinogenic properties of curcumin continue to be a subject of great interest. Evidence that it can inhibit the initiation, progression, and continued survival of cancerous cells, likewise, continues to accumulate [8]. Curcumin inhibits cell proliferation by interfering with the cell cycle and inducing apoptosis in colorectal carcinoma cells $[9,10]$. Curcumin also has chemopreventive potential for colorectal cancer as seen in a mouse model and in human clinical trials $[4,8,11,12]$.

Despite promising findings, curcumin has yet to be approved as an effective chemotherapeutic agent. Testing in animal models and human clinical trials has revealed that the bioavailability of curcumin is low, owing to its poor absorption across the gut, limited tissue distribution, rapid metabolism, and its subsequent elimination from the body [13]. In light of these findings, numerous strategies have been devised to address the limitations of curcumin, including the design and synthesis of novel structural analogues [14]. One such compound is GOY030 [15] and two other compounds, FLLL-11 and FLLL12 , which were synthesized by our laboratories. In our present study, we compared the inhibitory efficacy of GOY030, FLLL-11, FLLL-12, and curcumin in human colorectal cancer cell lines. We demonstrated that GO-Y030, FLLL-11, and FLLL-12 are more active than curcumin in the inhibition of cell proliferation and induction of PARP and caspase- 3 cleavages in all three colorectal cancer cell lines. GO-Y030 appears to be slightly more potent than FLLL-11 and FLLL-12 in these colorectal cancer cell lines.
Therefore, the synthetic derivatives of curcumin studied have potential as new therapeutic agents for colorectal cancer.

\section{Methods \\ Cell Culture}

Human colorectal cancer cells, HCT-116, SW480, and HT29, WI-38 human lung fibroblasts, and MCF-10A immortalized human mammary epithelial cells were acquired from the American Type Culture Collection (ATCC). The cancer cells were maintained in $1 \times$ Dulbecco's Modification of Eagle's Medium (DMEM) supplemented with 10\% fetal bovine serum (FBS) (Invitrogen), $4.5 \mathrm{~g} / \mathrm{L} \mathrm{L}$ glutamine and sodium pyruvate (Mediatech), and $1 \%$ Penicillin/Streptomycin $(\mathrm{P} / \mathrm{S})$. The cells were kept in a humidified $37^{\circ} \mathrm{C}$ incubator and aired with $5 \% \mathrm{CO}_{2}$. The MCF-10A cells were maintained in Ham's F12 media (Mediatech) supplemented with 5\% FBS, $5 \mu \mathrm{g} / \mathrm{ml}$ insulin, $1 \mu \mathrm{g} / \mathrm{ml}$ hydrocortisone, $10 \mu \mathrm{g} / \mathrm{ml}$ epidermal growth factor (EGF), $100 \mu \mathrm{g} / \mathrm{ml}$ cholera toxin, and $1 \% \mathrm{P} / \mathrm{S}$. Normal human bladder smooth muscle cells (HdSMC) were purchased from Lonza (Walkersville, MD) and Normal human colonic smooth muscle cells (HCSMC) were purchased from ScienCell (Carlsbad, CA).

\section{Western blot analysis}

HCT-116, SW480, and HT-29 colorectal cancer cells, WI38, HdSMC, HCSMC, and MCF-10A cells were treated with the listed concentrations of GO-Y030, FLLL-11, FLLL-12, and Curcumin (Sigma-Aldriich, St. Louis, MO) for 24 hours. Total protein lysates $(50 \mu \mathrm{g} / \mathrm{lane})$, as determined by BCA Protein Assay Kit (Thermo Fisher Scientific, Rockford, IL), were separated by $10 \%$ SDS-polyacrylamide gels and transferred to membranes. Membranes were blotted with a 1:1000 dilution of antibodies against cleaved Poly (ADP-ribose) polymerase (PARP) (Asp214) and cleaved caspase-3 (Asp175) (Cell Signaling Technology, Beverly, MA). A 1:1000 dilution of anti-GAPDH (glyceraldehydes-3-phosphoate dehydrogenase) monoclonal antibody (MAB374, Chemicon International, Inc., Temecula, CA) was used as a protein loading control. The blots were incubated with a 1:600 dilution of secondary fluorescein-linked anti-mouse or anti-rabbit antibody followed by incubation with a 1:2500 dilution of alkaline phosphatase conjugated anti-fluorescein antibody (Amersham Biosciences, Piscataway, NJ). Blots were scanned with ImageQuant software using an ECF Western blotting detection system (Amersham Biosciences, Piscataway, NJ) on a Molecular Dynamics Storm PhosphorImager (Sunnyvale, CA). The fluorescent signals were scanned and documented using a Storm 860 scanner (Molecular Dynamics, Sunnyvale, CA, USA). 


\section{MTT Cell Viability Assay}

HCT-116, SW480, and HT-29 human colorectal cancer cells and WI-38 lung fibroblasts were seeded in 96-well plates (4000 cells/well) in DMEM with $10 \%$ FBS. The following day the cells were treated with GO-Y030, FLLL-11, FLLL-12, and Curcumin (Sigma-Aldriich, St. Louis, MO) as indicated and incubated for 72 hours. $25 \mu \mathrm{l}$ of MTT (Thiazolyl Blue Tetrazolium Bromide: M5655, SIGMA) was added to each well and incubated for 3.5 hours followed by the addition of $100 \mu \mathrm{l}$ of N,N-dimethylformamide (D4551, SIGMA) solubilization solution. The plates were left at room temperature overnight to allow complete lysis of the cells and read at $450 \mathrm{~nm}$ the following day. Half-Maximal inhibitory concentrations $\left(\mathrm{IC}_{50}\right)$, the drug concentration at which $50 \%$ growth inhibition is achieved, was calculated using Sigma Plot 9.0 software (Systat Software Inc., San Jose, CA) with the 4 parameter logistic function standard curve analysis for dose response.

\section{Bright Field Microscopy}

Colorectal cancer cells were seeded in six-well plates at a density of $4 \times 10^{4}$ cells per well and incubated overnight to allow the cells to adhere. The cells were treated with the listed agents and incubated for four days. The cells were washed briefly in PBS before being photographed using bright field microscopy (10× magnification). Images were taken with a Model 9.0 Monochrome-6 Camera on a computer equipped with Spot Advanced imaging software (Diagnostic Instruments Inc., Sterling Heights, MI). Three images of each treatment were taken from randomly chosen fields and a representative image was selected for display in the figure.

\section{Results}

New curcumin analogues are more potent than curcumin in inhibiting cell viability in human colorectal cancer cells In this study, we examined the growth suppressive activities of three structural analogues of curcumin, GO-Y030, FLLL-11, and FLLL-12 (Figures 1A and 1B), in three independent human colorectal cancer cell lines, HCT-116, SW480, and HT-29, as well as WI-38 lung fibroblasts. We first examined the inhibitory effect of cell viability by curcumin, GO-Y030, FLLL-11, and FLLL-12 in human colorectal cancer cell lines and WI-38 normal human lung fibroblasts. After 72 hours of treatment, the percent of inhibition was determined. GO-Y030, FLLL-11, and FLLL12 all exhibited greater levels of inhibition, when compared to untreated cells in colorectal cancer cell lines than curcumin (Figures 2A and 2B). Furthermore, FLLL-11 and FLLL-12 had a low inhibitory effect on cell viability in WI38 human lung fibroblasts (Figure 2C). Representative pictures of the inhibition of cell growth were taken for the three colorectal cancer cell lines, SW480 (Figure 2D), HCT116 and HT-29 (Data not shown). IC $_{50}$ values were
A

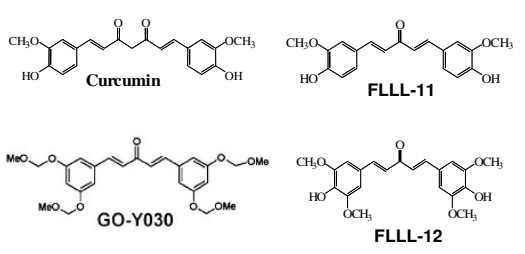

B

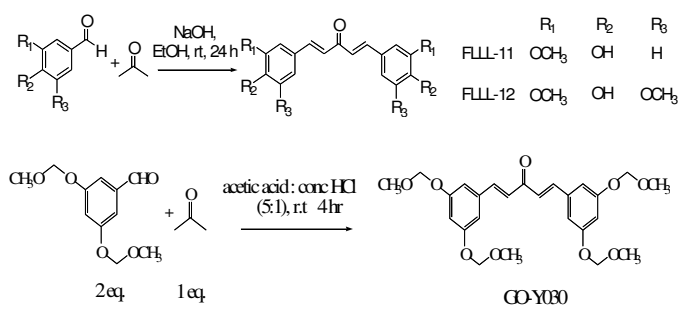

Figure I

(A) The chemical structures of curcumin, GO-Y030, FLLL- I I, and FLLL- I 2. (B) Synthesis of FLLL- I I, FLLL- I2, and GO-Y030.

calculated for each cell line and treatment (Table 1). The results demonstrate that GO-Y030, FLLL-11, and FLLL-12 are more potent than curcumin in the growth suppression of the human colorectal cancer cell lines studied.

\section{GO-Y030, FLLL-II, and FLLL-I 2 are more potent than curcumin in inducing apoptosis in colorectal cancer cells} The induction of apoptosis was examined using cleaved PARP (Asp214) and cleaved caspase-3 (Asp175) in HCT116, SW480, and HT-29 colorectal cancer cells. GOY030, FLLL-11, and FLLL-12 at 2.5 and $5 \mu \mathrm{M}$ induced cleaved PARP and cleaved caspase- 3 in HCT116 colorectal cancer cells as evidenced by increased levels of cleaved PARP and cleaved caspase- 3 while 10 and $20 \mu \mathrm{M}$ of curcumin induced increased levels of cleaved PARP and caspase-3 (Figure 3A). HT-29 colorectal cancer cells seem to be the most resistance to curcumin and these three analogues, $5 \mu \mathrm{M}$ of GO-Y030, FLLL-11, and FLLL-12 induced increased levels of cleaved PARP, whereas 10 and $20 \mu \mathrm{M}$ of curcumin did not exhibit increased levels of cleaved PARP (Figure 3B). $5 \mu \mathrm{M}$ of GO-Y030 and $20 \mu \mathrm{M}$ of curcumin induced increased levels of cleaved caspase-3 while slightly increased of cleaved caspase-3 was detected in FLLL-11 and FLLL-12 treated HT-29 cells (Figure 3B). 20 $\mu \mathrm{M}$ of curcumin induced increased levels of cleaved PARP (Figure 3C). FLLL-12 at 5 and GO-Y030 at 2.5 and $5 \mu \mathrm{M}$ induced increased levels of cleaved PARP and caspase- 3 in SW480 colorectal cancer cells (Figure 3C). $20 \mu \mathrm{M}$ of curcumin induced slightly increased levels of cleaved PARP and caspase-3 in SW480 cancer cells (Figure 3C). The results indicate that GO-Y030, FLLL-11, and FLLL-12 are more potent than curcumin in inducing PARP and caspase-3 cleavages in the three human colorectal cancer cell lines studied. Further, GO-Y030, FLLL-11 and FLLL-12 
A
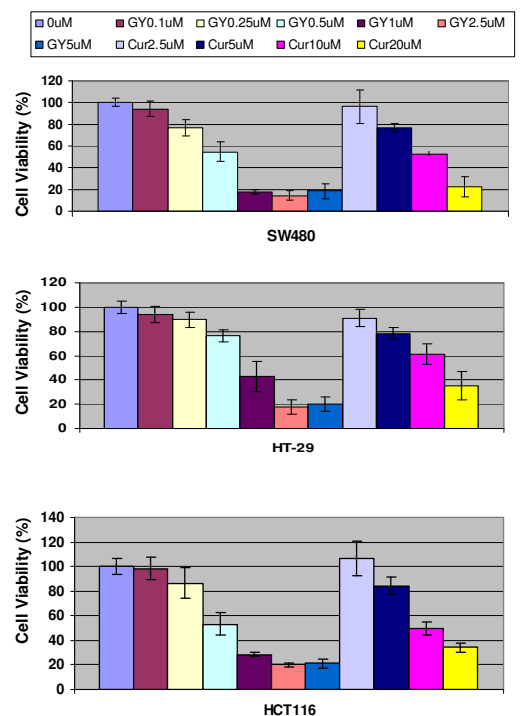

D
B

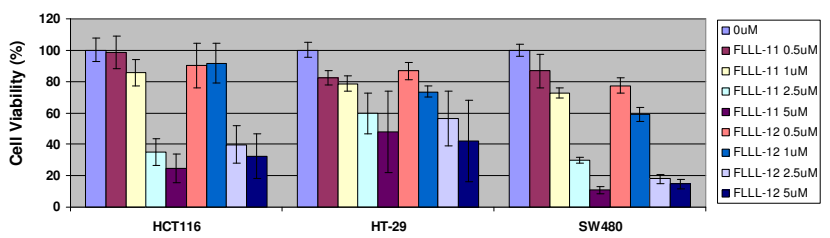

C

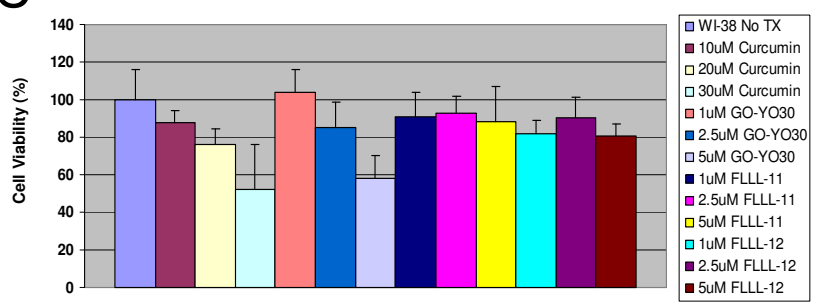

Untreated

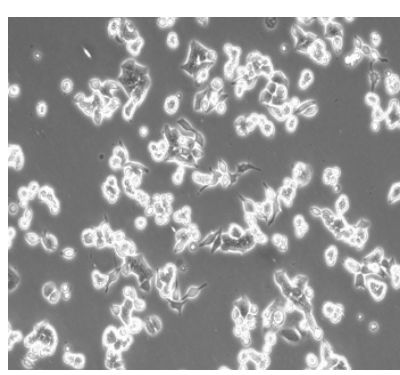

GO-YO30 $5 \mu \mathrm{M}$

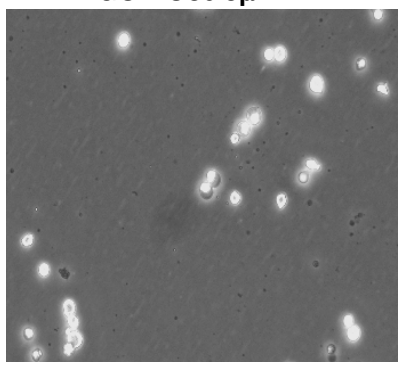

DMSO

FLLL-11 5 $\mu \mathrm{M}$

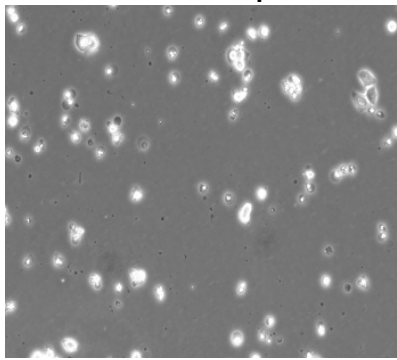

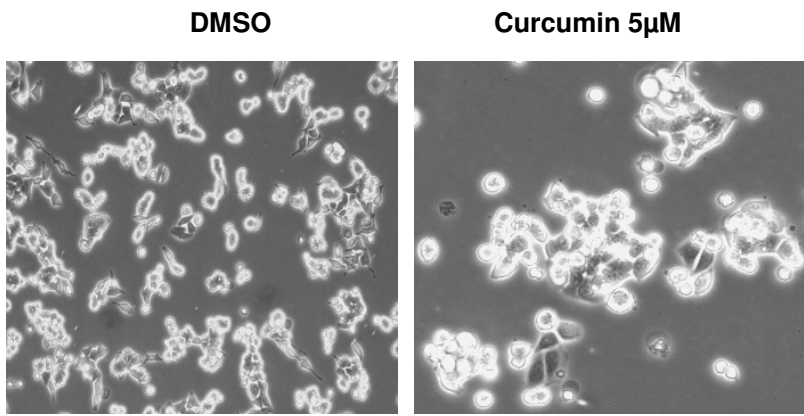

SW480

FLLL-12 5 $\mu \mathrm{M}$

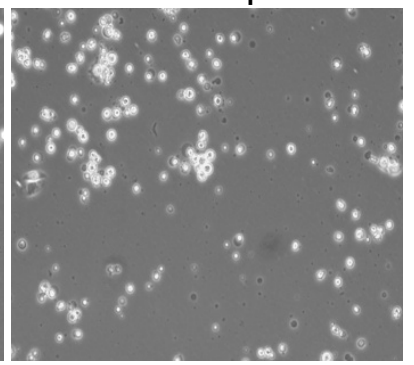

Figure 2

The inhibitory effects of cell viability by (A) curcumin (Cur) and GO-Y030 (GY), (B) FLLL-I I and FLLL-I 2 in human colorectal cancer cell lines, HCTI I6, HT-29, SW480, and (C) WI-38 normal human lung fibroblasts. Representative pictures in (D) SW480 colon cancer cells were shown. 
Table I: The IC50 $(\mu \mathrm{M})$ of curcumin and its analogues, GO-Y030, FLLL- II, and FLLL- 12 in human colon cancer cells and WI-38 human lung fibroblasts

\begin{tabular}{lcccc}
\hline & $\begin{array}{c}\mathrm{HCTII6} \\
(\mathrm{IC50} \mu \mathrm{M})\end{array}$ & $\begin{array}{c}\mathrm{HT}-29 \\
(\mathrm{IC} 50 \mu \mathrm{M})\end{array}$ & $\begin{array}{c}\text { SW480 } \\
(\mathrm{IC5} 0 \mu \mathrm{M})\end{array}$ & $\begin{array}{c}\mathrm{WI}-38 \\
(\mathrm{IC5} 5 \mu)\end{array}$ \\
\hline Curcumin & 10.91 & 13.31 & 10.26 & 48.82 \\
GO-Y030 & $0.6 \mathrm{I}$ & 0.96 & 0.51 & 13.78 \\
FLLL-II & 2.07 & 4.48 & 1.64 & $>1000.00$ \\
FLLL-12 & 2.49 & 3.35 & 1.17 & $>1000.00$ \\
\hline
\end{tabular}

*Cancer cells were treated for 72 hours and cell viability was determined by MTT assays and $\mathrm{IC}_{50}$ values were analyzed.

appear to induce PARP cleavages in normal human colonic smooth muscle cells. However, GO-Y030, FLLL11, and FLLL-12 did not induce PARP cleavages in WI-38 normal human lung fibroblasts, normal human bladder smooth muscle cells, and MCF-10A non-malignant human mammary epithelial cells (Figures 3D and 3E).

\section{Discussion}

Current anticancer therapies tend to be inadequate in terms of their therapeutic efficacy and the undesirable side effects that they produce. Bioactive compounds, known as phytochemicals, have been found to exhibit growth-suppressive activity as well as chemopreventive properties against various types of cancer [2]. Curcumin is one of the most widely characterized phytochemicals and is the active ingredient in the rhizome of the plant turmeric (Curcuma longa). Curcumin has both antioxidant and anti-inflammatory properties $[2,3,16]$. It has also been shown to protect against carcinogenesis and in the prevention of tumor formation and development in several cancer types. In a variety of animal tumor models, curcumin has been found to suppress angiogenesis and metastasis [2-6,17-21]. The growth suppressive activity and bioavailability of curcumin in humans may be effective as a preventive agent; however, it may not be sufficient as a therapeutic agent in colorectal cancer. Analogues of curcumin, which exhibit increased potency, are needed as more effective therapeutic agents for colorectal cancer treatments.

There are several curcumin analogues that have already been reported, such as dimethoxycurcumin and EF-24 [22-26]. We compared the inhibitory efficacy of new curcumin analogues, GO-Y030, FLLL-11, and FLLL-12 in colorectal cancer cell lines. We demonstrated that GOY030, FLLL-11, and FLLL-12 are more potent than curcumin in the inhibition of cell viability/proliferation in all three independent human colorectal carcinoma cell lines studied. GO-Y030, FLLL-11, and FLLL-12 are also more potent than curcumin in the induction of cleaved PARP, evidence of apoptosis, in the colorectal cancer cells studied. Although, cleaved PARP was also observed in normal colonic smooth muscle cells by GO-Y030, FLLL-11, and FLLL-12, GO-Y030, FLLL-11, and FLLL-12 did not induce cleaved PARP in WI-38 normal human lung fibroblasts, normal bladder smooth muscle cells, and MCF-10A immortalized, non-malignant, human mammary epithelial cells. FLLL-11 and FLLL-12 appear to be slightly less potent than GO-Y030 in the inhibition of cell viability in the colorectal cancer cell lines studied. However, FLLL-11 and FLLL-12 have less inhibitory effects than GO-Y030 in WI-38 normal human lung fibroblasts. FLLL-11 and FLLL12 are respectively, 223 and 298 fold times more toxic in colorectal cancer cells than in WI-38 normal human lung fibroblasts compared to GO-Y030 which is only 14 fold times more toxic. Curcumin has been found to be safe in clinical trials and dose-limiting toxicity was not observed [27-30]. Similar to curcumin, FLLL-11 and FLLL-12 seem to have low toxicity in WI-38 normal human lung fibroblasts, normal bladder smooth muscle cells and non-malignant MCF-10A mammary epithelial cells except FLLL-11, FLLL-12, and GO-Y030 induce cleaved PARP in normal colonic smooth muscle cells. It will be interested to evaluate the inhibitory efficacy of FLLL-11, FLLL-12, and GOY030 to suppress the growth of the human colon tumor cells in mouse model in vivo. Further, it will also be necessary to evaluate the possible toxicity of FLLL-11, FLLL-12, and GO-Y030 comparing to curcumin in mice without tumors in vivo in particular in colon to verify the toxicity we have observed in normal colonic smooth muscle cells.

To evaluate the possible drug-likeness of these curcumin analogues, we used QikProp (Schrodinger LLC) to compute the absorption, distribution, metabolism, excretion and toxicity (ADME/Tox) properties of curcumin, FLLL11, FLLL-12 and GO-Y030. Overall, fifty "drug-likeness" parameters have been calculated, covering molecular weight, polarity, solubility, cell permeability, blood brain barrier, HERG $\mathrm{K}^{+}$blockage, HSA binding and metabolic stability, etc. For curcumin itself, the prediction shows concern on potential HERG $\mathrm{K}^{+}$channel blockage, and more seriously a very poor cell permeability index for both Caco-2 and MDCK tests. The data seem to be consistent with the widely reported poor bioavailability of curcumin. FLLL-11, FLLL-12 and GO-Y030 all have favorable drug-likeness profiles. However, the relatively minor concerns are: 1) FLLL-11 has subpar cell permeability indexes (Caco2: 485; MDCK: 226), not as good as FLLL-12 (Caco2: 1164; MDCK: 583) and GO-Y030 (Caco2: 3073; MDCK: 1665); 2) the $\mathrm{IC}_{50}$ HERG $\mathrm{K}^{+}$blockage index for all three compounds are on borderline as all three compounds have QPlogHERG values around -5.5, below the 5 redline. Optimization to lessen this seems needed; 3 ) GO-Y030 has poor oral absorption index (index as 1 for low absorption) compared to FLLL-11 and FLLL-12 (both indexes as 3 for high oral absorption). In addition, most indicators show that GO-Y030 has the more deviations 
A
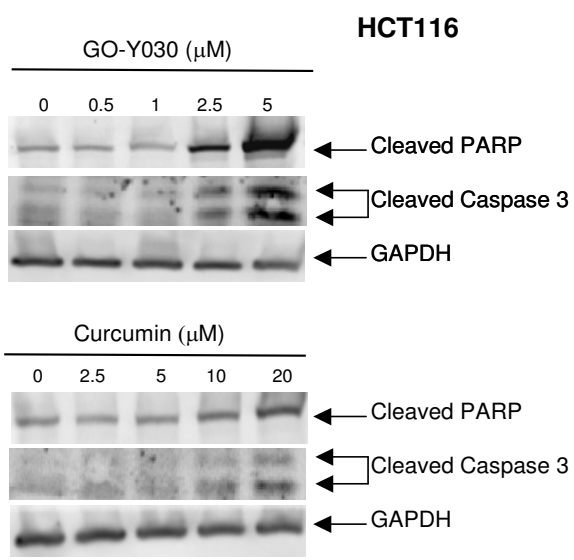

FLL-11( $\mu \mathrm{M}) \quad$ FLLL-12 $(\mu \mathrm{M})$
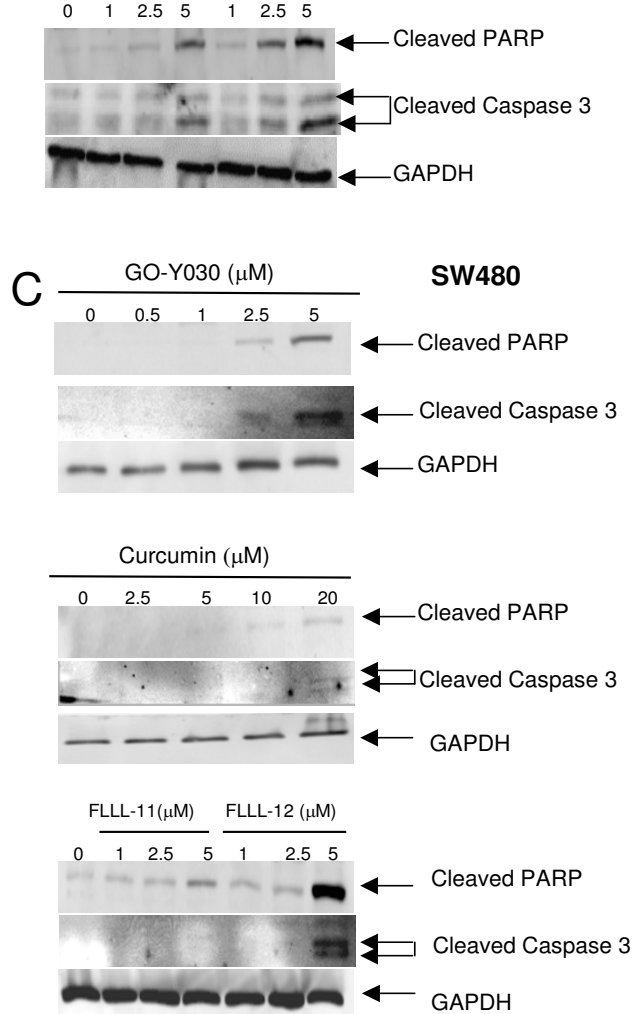

B
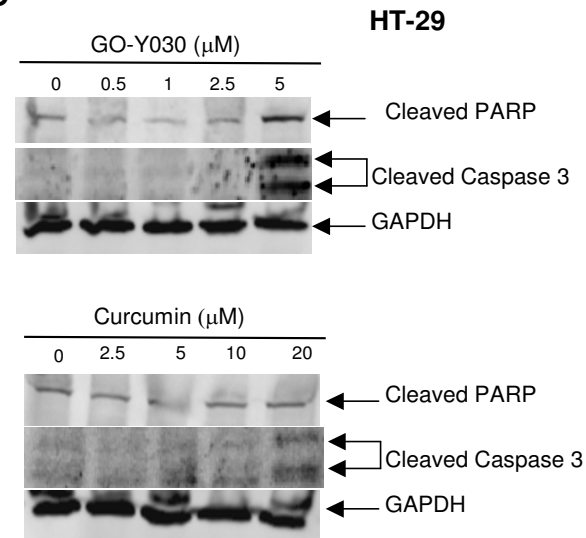

$\frac{\mathrm{FLLL}-11(\mu \mathrm{M})}{12.5 \quad 5} \frac{\mathrm{FLLL}-12(\mu \mathrm{M})}{12.5 \quad 5}$

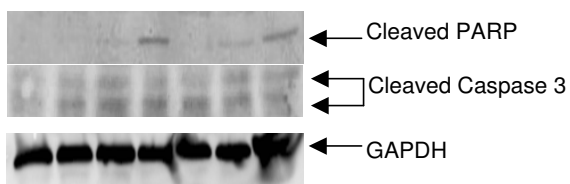

D
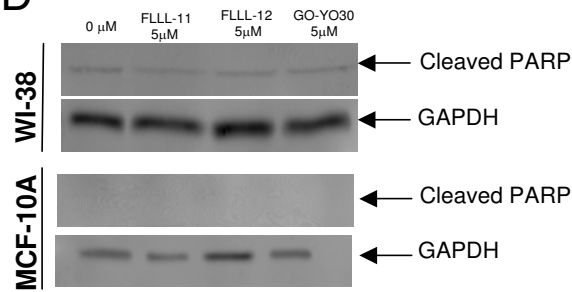

$E$
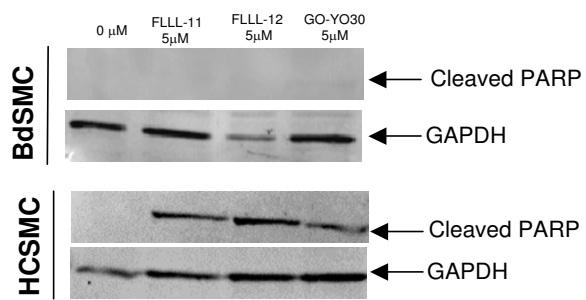

Figure 3

Curcumin and its analogues, GO-Y030, FLLL-I I, and FLLL- 12 induced PARP and/or caspase-3 cleavages in (A) HCTI I6, (B) HT-29, (C) SW480 human colorectal cancer cells, (D) WI-38 and MCF-IOA cells, (E) normal human and colonic bladder smooth muscle cells. Colorectal cancer cells and normal human cells were treated for 24 hours. Western blot was used to detect the PARP cleavages using antibodies against cleaved PARP and cleaved caspase-3 (Cell Signaling Technology, Beverly, MA). The same membranes were analyzed with a I:I000 dilution of anti-GAPDH monoclonal antibody (Chemicon International, Inc., Temecula, CA). 
from existing drugs than FLLL-11 and FLLL-12. Overall evaluations show that FLLL-12 has the closest drug-likeness profile and has $86 \%$ similarity to propafenone and trimethobenzamide averaged over all fifty parameters. Therefore, it will be of interest to evaluate the inhibitory efficacy of these curcumin analogues in tumor model in vivo and in vivo pharmacokinetics in the future.

\section{Conclusion}

Our results demonstrated that the curcumin analogues, GO-Y030, FLLL-11, and FLLL-12 are more potent than curcumin in the inhibition of cell proliferation and in the induction of apoptosis in three different human colorectal cancer cells. GO-Y030, FLLL-11, and FLLL-12 pass the drug-likeness test, with FLLL-12 having the highest druglikeness. Therefore, GO-Y030, FLLL-11, and FLLL-12 have the translational potential as novel cancer therapeutic or preventive agents for human colorectal carcinoma and deserve the further evaluation.

\section{Abbreviations}

$\mathrm{IC}_{50}$ : Half-Maximal inhibitory concentrations; MTT: 3(4,5-dimethylthiazol-2-yl)-2,5-diphenyltetrazolium bromide; PARP: Poly (ADP-ribose) polymerase; GAPDH: Glyceraldehyde-3-phosphate dehydrogenase; PK/PD: Pharmacokinetics/Pharmacodynamics.

\section{Competing interests}

The authors declare that they have no competing interests.

\section{Authors' contributions}

LC participated in experiment designs, conducted most of the experiments, contributed to the analysis and interpretation of data, and drafted the manuscript. BH and SD worked on the pictures of the treated colorectal cancer cells and contributed to the manuscript preparation. SB worked on the effects on normal human cells. CC participated in Western blots analysis. CL provided the information of drug-likeness. P-K L and J F synthesized and provided the curcumin analogues in collaboration. All authors read and approved the final manuscript.

\section{Acknowledgements}

This work was supported in part by a National Foundation for Cancer Research and Elsa U. Pardee grants given to Jiayuh Lin. We thank to Dr. Hiroyuki Shibata at the Institute of Development, Aging and Cancer at Tohoku University for providing GO-Y030.

\section{References}

I. Gatta G, Ciccolallo L, Capocaccia R, Coleman MP, Hakulinen T, Møller H, Berrino F: Differences in colorectal cancer survival between European and US populations: the importance of sub-site and morphology. European Journal of Cancer 2003, 39(I5):2214-2222.

2. Aggarwal B, Shishodia S: Molecular targets of dietary agents for prevention and therapy of cancer. Biochem Pharmacol 2006, 7I:1397-|42I.
3. Hanif R, Qiao L, Shiff SJ, Rigas B: Curcumin, a natural plant phenolic food additive, inhibits cell proliferation and induces cell cycle changes in colon adenocarcinoma cell lines by a prostaglandin-independent pathway. I Lab Clin Med 1997, 130(6):576-584.

4. Kawamori T, Lubet R, Steele VE, Kelloff GJ, Kaskey RB, Rao CV, Reddy BS: Chemopreventive effect of curcumin, a naturally occurring anti-inflammatory agent, during the promotion/ progression stages of colon cancer. Cancer research 1999, 59(3):597-60I.

5. Azuine MA, Bhide SV: Chemopreventive effect of turmeric against stomach and skin tumors induced by chemical carcinogens in Swiss mice. Nutr Cancer 1992, I7(1):77-83.

6. Azuine MA, Bhide SV: Protective single/combined treatment with betel leaf and turmeric against methyl (acetoxymethyl) nitrosamine-induced hamster oral carcinogenesis. Int J Cancer 1992, 5 I (3):412-415.

7. Goel A, Kunnumakkara AB, Aggarwal BB: Curcumin as "Curecumin": from kitchen to clinic. Biochem Pharmacol 2008, 75(4):787-809.

8. Hatcher H, Planalp R, Cho J, Torti FM, Torti SV: Curcumin: From ancient medicine to current clinical trials. Cell Mol Life Sci 2008, 65(II):163I-52.

9. Chaudhary LR, Hruska KA: Inhibition of cell survival signal protein kinase B/Akt by curcumin in human prostate cancer cells. J Cell Biochem 2003, 89(1): I-5.

10. Narayan S: Curcumin, a multi-functional chemopreventive agent, blocks growth of colon cancer cells by targeting betacatenin-mediated transactivation and cell-cell adhesion pathways. Journal of molecular histology 2004, 35(3):30I-307.

II. Kelloff GJ, Crowell JA, Steele VE, Lubet RA, Boone CW, Malone WA, Hawk ET, Lieberman R, Lawrence JA, Kopelovich L, et al.: Progress in cancer chemoprevention. Annals of the New York Academy of Sciences 1999, 889:1-13.

12. Kelloff GJ, Crowell JA, Steele VE, Lubet RA, Malone WA, Boone CW, Kopelovich L, Hawk ET, Lieberman R, Lawrence JA, et al:: Progress in cancer chemoprevention: development of diet-derived chemopreventive agents. The Journal of nutrition 2000, I30(2S Suppl):467S-47IS.

13. Anand P, Kunnumakkara AB, Newman RA, Aggarwal BB: Bioavailability of curcumin: problems and promises. Mol Pharm 2007, 4(6):807-818.

14. Mosley CA, Liotta DC, Snyder JP: Highly active anticancer curcumin analogues. Adv Exp Med Biol 2007, 595:77-103.

15. Ohori H, Yamakoshi H, Tomizawa M, Shibuya M, Kakudo Y, Takahashi A, Takahashi S, Kato S, Suzuki T, Ishioka C, et al:: Synthesis and biological analysis of new curcumin analogues bearing an enhanced potential for the medicinal treatment of cancer. Molecular cancer therapeutics 2006, 5(10):2563-257I.

16. Shi M, Cai Q, Yao L, Mao Y, Ming Y, Ouyang G: Antiproliferation and apoptosis induced by curcumin in human ovarian cancer cells. Cell Biol Int 2006, 30(3):22I-226.

17. Aggarwal BB, Shishodia S, Takada Y, Banerjee S, Newman RA, BuesoRamos CE, Price JE: Curcumin suppresses the paclitaxelinduced nuclear factor-kappaB pathway in breast cancer cells and inhibits lung metastasis of human breast cancer in nude mice. Clin Cancer Res 2005, I I(20):7490-7498.

18. Bachmeier B, Nerlich AG, lancu CM, Cilli M, Schleicher E, Vene R, Dell'Eva R, Jochum M, Albini A, Pfeffer U: The chemopreventive polyphenol Curcumin prevents hematogenous breast cancer metastases in immunodeficient mice. Cell Physiol Biochem 2007, 19(I-4): I37-I52.

19. Ikezaki S, Nishikawa A, Furukawa F, Kudo K, Nakamura H, Tamura K, Mori $\mathrm{H}$ : Chemopreventive effects of curcumin on glandular stomach carcinogenesis induced by $\mathbf{N}$-methyl-N'-nitro- $\mathbf{N}$ nitrosoguanidine and sodium chloride in rats. Anticancer Res 200I, 2 I (5):3407-34II.

20. Frank N, Knauft J, Amelung F, Nair J, Wesch H, Bartsch H: No prevention of liver and kidney tumors in Long-Evans Cinnamon rats by dietary curcumin, but inhibition at other sites and of metastases. Mutat Res 2003, 523-524: I27-135.

21. Gururaj AE, Belakavadi M, Venkatesh DA, Marme D, Salimath BP: Molecular mechanisms of anti-angiogenic effect of curcumin. Biochem Biophys Res Commun 2002, 297(4):934-942.

22. Tamvakopoulos C, Dimas K, Sofianos Z, Hatziantoniou S, Han Z, Liu $Z$, Wyche J, Pantazis P: Metabolism and anticancer activity of 
the curcumin analogue, dimethoxycurcumin. Clin Cancer Res 2007, I 3: | 269- I 277.

23. Ohtsu H, Xiao Z, Ishida J, Nagai M, Wang H, Itokawa H, Su C, Shih C, Chiang T, Chang E, et al.: Antitumor agents. 217. Curcumin analogues as novel androgen receptor antagonists with potential as anti-prostate cancer agents. J Med Chem 2002, 45:5037-5042.

24. Mosley C, Liotta D, Snyder J: Highly active anticancer curcumin analogues. Adv Exp Med Biol 2007, 595:77- 103.

25. Youssef D, Nichols C, Cameron T, Balzarini J, De Clercq E, Jha A: Design, synthesis, and cytostatic activity of novel cyclic curcumin analogues. Bioorg Med Chem Lett 2007, 17:5624-5629.

26. Youssef KM, El-Sherbeny MA, El-Shafie FS, Farag HA, Al-Deeb OA, Awadalla SA: Synthesis of curcumin analogues as potential antioxidant, cancer chemopreventive agents. Arch Pharm (Weinheim) 2004, 337(1):42-54.

27. Aggarwal B, Kumar A, Bharti A: Anticancer potential of curcumin: preclinical and clinical studies. Anticancer Res 2003, 23:363-398.

28. Sharma R, Euden S, Platton S, Cooke D, Shafayat A, Hewitt H, Marczylo T, Morgan B, Hemingway D, Plummer S, et al.: Phase I clinical trial of oral curcumin: biomarkers of systemic activity and compliance. Clin Cancer Res 2004, 10:6847-6854.

29. Hsu C, Cheng A: Clinical studies with curcumin. Adv Exp Med Biol 2007, 595:425-45I.

30. Hanai H, lida T, Takeuchi K, Watanabe F, Maruyama Y, Andoh A, Tsujikawa T, Fujiyama Y, Mitsuyama K, Sata M, et al:: Curcumin maintenance therapy for ulcerative colitis: randomized, multicenter, double-blind, placebo-controlled trial. Clin Gastroenterol Hepatol 2006, 4:1502-1506.

\section{Pre-publication history}

The pre-publication history for this paper can be accessed here:

http://www.biomedcentral.com/1471-2407/9/99/prepub http://www.biomedcentral.com/1471-2407/9/99 